\title{
GLOBAL SOLUTION TO A PHASE FIELD MODEL WITH IRREVERSIBLE AND CONSTRAINED PHASE EVOLUTION
}

\author{
BY \\ FABIO LUTEROTTI (Dipartimento di Matematica, Università di Brescia, via Branze 38, 25123 \\ Brescia, Italy), \\ GIULIO SCHIMPERNA (Dipartimento di Matematica, Università di Pavia, via Ferrata 1, 27100 \\ Pavia, Italy), \\ AND \\ ULISSE STEFANELLI (Dipartimento di Matematica, Università di Pavia, via Ferrata 1, 27100 \\ Pavia, Italy)
}

\begin{abstract}
This note deals with a nonlinear system of PDEs describing some irreversible phase change phenomena that account for a bounded limit velocity of the phase transition process. An existence result is established by using time discretization, compactness arguments, and techniques of subdifferential operators.
\end{abstract}

1. Introduction. The present analysis is concerned with a nonlinear system of partial differential equations describing irreversible phase change phenomena with bounded velocity of phase transition. Such a system comes from a recent model, proposed by Frémond, which relies on the consideration that the movements of the microscopic particles may affect the macroscopic phase transition process as well. In particular, our system is to be regarded in the more general framework of phase field models. The literature concerning this kind of model is rather wide and the reader is referred, e.g., to [6], [7] and the references therein for a detailed analysis.

Here we want to recall the main features of this model, whose derivation was first given in [3]. Let us consider a two-phase substance contained in a domain $\Omega \subset \mathbb{R}^{3}$ and a given final time $T>0$. We choose the volume fraction of one of the phases as a state quantity and denote it by $\chi=\chi(x, t)$, for $x \in \Omega$ and $t \in[0, T]$. Hence, $\chi$ satisfies the relation $0 \leq \chi \leq 1$ and, assuming that no voids appear in the mixture, the volume fraction of the other phase is simply given by $1-\chi$. The absolute temperature $\theta=\theta(x, t)$ is the other

Received June 19, 2000.

2000 Mathematics Subject Classification. Primary 80A22, 35K55, 47H05.

Key words and phrases. Phase field models, microscopic movements, maximal monotone graphs, doubly nonlinear evolution systems.

E-mail address: luterott@bsing.ing.unibs.it

E-mail address: schimper@dragon.ian.pv.cnr.it

E-mail address: ulisse@dimat.unipv.it 
state variable for the thermodynamical system and it has to be nonnegative. Taking into account the power of the microscopic movements, we address the following energy balance equation:

$$
\partial_{t} e+\operatorname{div} \mathbf{q}=B \partial_{t} \chi+\mathbf{H} \cdot \nabla \partial_{t} \chi
$$

where $e$ denotes the internal energy, $\mathbf{q}=-k \nabla \theta$ stands for the heat flux vector (i.e., the Fourier law is assumed with constant conductivity $k$ ), and $B$ and $\mathbf{H}$ are, respectively, a scalar quantity and a vector resulting from the microscopic interior forces. A primer relation between the quantities $B$ and $\mathbf{H}$ comes from an application of the virtual power principle. Namely, in the absence of external actions we readily get

$$
-\operatorname{div} \mathbf{H}+B=0 .
$$

Now, we choose the free energy $\Psi$ as

$$
\Psi(\chi, \nabla \chi, \theta)=-c_{s} \theta \log \theta-\frac{L}{\theta_{c}}\left(\theta-\theta_{c}\right) \chi+I_{[0,1]}(\chi)+\frac{\nu}{2}|\nabla \chi|^{2},
$$

where $L>0$ stands for the latent heat at the critical transition temperature $\theta_{c}>0$, $c_{s}>0$ represents the specific heat, the parameter $\nu>0$ is the factor of the interfacial energy term, and $I_{[0,1]}$ (i.e., the indicator function of $[0,1]$ ) plays the role of the constraint for the phase proportion $\chi$. Let us point out that (1.3) is the standard choice for the phase field models with constraint on $\chi$. Moreover, we set

$$
\Phi\left(\partial_{t} \chi\right)=\frac{\mu}{2}\left(\partial_{t} \chi\right)^{2}+I_{[0,+\infty)}\left(\partial_{t} \chi\right)
$$

for the pseudo-potential of dissipation, where $\mu$ is a positive coefficient. Note that the above position accounts for the irreversibility of the phase change because of the presence of the term $I_{[0,+\infty)}\left(\partial_{t} \chi\right)$.

Our choices for the constitutive laws for $B$ and $\mathbf{H}$ are provided by

$$
B=\frac{\partial \Psi}{\partial \chi}+\frac{\partial \Phi}{\partial\left(\partial_{t} \chi\right)}, \quad \mathbf{H}=\frac{\partial \Psi}{\partial(\nabla \chi)},
$$

while the internal energy $e$ is related to the free energy $\Psi$ and to the entropy $s=-\partial \Psi / \partial \theta$ by the rather classical relationship

$$
e=\Psi+s \theta=\Psi-\theta \frac{\partial \Psi}{\partial \theta}
$$

Actually, it is not difficult to show [8] that in this setting the Second Principle of Thermodynamics is satisfied in the form of the Clausius-Duhem inequality. The balance and constitutive laws - together with (1.3) and (1.4)-yield the following system:

$$
\begin{gathered}
c_{s} \partial_{t} \theta+L \partial_{t} \chi-k \Delta \theta=-\frac{L}{\theta_{c}}\left(\theta-\theta_{c}\right) \partial_{t} \chi+\mu\left(\partial_{t} \chi\right)^{2}+\zeta \partial_{t} \chi, \\
\mu \partial_{t} \chi-\nu \Delta \chi+\zeta+\eta=\frac{L}{\theta_{c}}\left(\theta-\theta_{c}\right),
\end{gathered}
$$

where

$$
\eta \in \partial I_{[0,1]}(\chi) \text { and } \zeta \in \partial I_{[0,+\infty)}\left(\partial_{t} \chi\right)
$$

almost everywhere in the space-time domain. We have denoted by $\partial I_{[0,1]}\left(\operatorname{resp} . \partial I_{[0,+\infty)}\right)$ the subdifferential of $I_{[0,1]}\left(\right.$ resp. $\left.I_{[0,+\infty)}\right)$. 
As one can see at once, the full model (1.7)-(1.9) looks very difficult to handle, even after noticing that $\zeta \partial_{t} \chi$ vanishes in (1.7), thanks to the definition of $\partial I_{[0,+\infty)}\left(\partial_{t} \chi\right)$. As far as we know, no existence result has yet been obtained for any initial-boundary problem related to the whole system (1.7)-(1.9). Indeed, we are able to mention only investigations devoted to some-more or less-modified versions of such a full model.

First, the paper [3] addresses the case when the right-hand side of (1.7) is negligible if compared with the left-hand side quantities (i.e., within the small perturbation assumption: $\theta$ close to $\theta_{c}$ and $\left.\mu\left(\partial_{t} \chi\right)^{2} \approx 0\right)$. Actually, in [3], the relation

$$
c_{s} \partial_{t} \theta+L \partial_{t} \chi-k \Delta \theta=0
$$

is considered. The latter is the standard expression of the energy balance equation in phase transition phenomena with Fourier heat flux law (see, e.g., [6]). An existence result is established for a Cauchy-Neumann problem by introducing a suitable regularization procedure together with a time discretization and deriving some careful a priori estimates. Such a result also holds in the limit case $\mu=0$.

In [4], the same authors consider the fully nonlinear energy balance equation (1.7), coupling it with an inclusion similar to (1.8), where anyway a dissipation term is added and only the reversible case is considered. A local existence theorem is then established for the resulting system, by using first a regularization procedure and then a fixed point technique that exploits the dissipation effects through the gain of regularity on $\partial_{t} \chi$. The problem addressed in [4] turns out to admit a global solution in the one-dimensional setting [13].

In [12] a further step is done in the direction of dealing with the whole system (1.7)(1.9), considering only $\mu\left(\partial_{t} \chi\right)^{2} \approx 0$ and allowing the temperature $\theta$ to be far from the critical value $\theta_{c}$. An existence result is obtained via a regularization-a priori estimatespassage to the limit procedure; it must be noted that the role of the irreversibility is crucial in the proofs, since it allows the use of maximum principle arguments.

On the other hand, in [9], these authors, together with Pierluigi Colli, are able to show an existence result for a system consisting of (1.7) and a modification of (1.8), where the term $-\Delta \chi$ is missing (it corresponds to setting $\nu=0$ in (1.8)). The latter is the typical inclusion of the phase relaxation model with irreversible evolution (introduced in [10]). The proof is performed by introducing a regularization procedure combined with a truncation. The derivation of some bounds of the unknowns is a key step and is obtained via the maximum principle and monotonicity arguments.

Unfortunately, we are still not able to give a direct answer to the analytical problems arising from (1.7)-(1.9). However, we remark that, from the experimental point of view, it is reasonable to assume that the phase change velocity $\partial_{t} \chi$ cannot exceed some finite natural limit $\lambda$. In this concern, we are led to consider a different class of constraints on $\partial_{t} \chi$ accounting for the limit velocity $\lambda$. A straightforward choice would be to replace $I_{[0,+\infty)}$ by $I_{[0, \lambda]}$ in (1.4). Clearly, this approach provides a good approximation of the above full model, when $\lambda$ is large enough. Moreover, we are aware of some strictly related situations where an $L^{\infty}$ a priori bound on $\partial_{t} \chi$ may be achieved ([9], [13]). Let us point out that the choice of the constraint $I_{[0, \lambda]}$ is still consistent with the Second Principle of Thermodynamics. 
Now, we follow the same steps of the derivation of the model and, taking into account the different constraint on $\partial_{t} \chi$, we obtain

$$
\begin{gathered}
c_{s} \partial_{t} \theta+L \partial_{t} \chi-k \Delta \theta=-\frac{L}{\theta_{c}}\left(\theta-\theta_{c}\right) \partial_{t} \chi+\mu\left(\partial_{t} \chi\right)^{2}+\xi \partial_{t} \chi \\
\mu \partial_{t} \chi-\nu \Delta \chi+\xi+\eta=\frac{L}{\theta_{c}}\left(\theta-\theta_{c}\right)
\end{gathered}
$$

where

$$
\eta \in \partial I_{[0.1]}(\chi) \text { and } \xi \in \partial I_{[0 . \lambda]}\left(\partial_{t} \chi\right)
$$

almost everywhere in the space-time domain. In this setting the boundedness of $\partial_{t} \chi$ is straightforward and turns out to be a very useful feature for the sake of an analytical investigation. On the other hand, the term $\xi \partial_{t} \chi$ does not vanish in (1.11), whence one has to deal with the further presence of a maximal monotone graph in the energy balance equation. Anyway, if we rewrite (1.11) as

$$
c_{s} \partial_{t} \theta-k \Delta \theta=\partial_{t} \chi\left(-\frac{L}{\theta_{c}} \theta+\mu \partial_{t} \chi+\xi\right),
$$

a simple comparison in (1.12) leads to

$$
c_{s} \partial_{t} \theta-k \Delta \theta=\partial_{t} \chi(\nu \Delta \chi-\eta-L)
$$

Thus, we can deal either with (1.14)-(1.12) or with the equivalent system (1.15)-(1.12). Let us also point out that more general classes of constraints could be admissible for the phase variable than those provided by (1.13). In particular, from a mathematical point of view, in this analysis we are able to deal with a general maximal monotone graph $\beta$ in place of the $\partial I_{[0,1]}$.

Clearly, for the purpose of a rigorous study, (1.12)-(1.14) have to be complemented with some initial and boundary conditions. We prescribe

$$
\begin{aligned}
& \theta(\cdot, 0)=\theta_{0}, \quad \chi(\cdot, 0)=\chi_{0} \quad \text { a.e. in } \Omega, \\
& \partial_{\mathbf{n}} \theta=\partial_{\mathbf{n}} \chi=0 \quad \text { a.e. on } \partial \Omega \times(0, T),
\end{aligned}
$$

where $\mathbf{n}$ stands for the outer unit normal vector to the boundary $\partial \Omega$. Actually, the boundary condition on $\chi$ seems to be natural and may be rigorously motivated in the framework of the derivation of the model. As for $\theta$, we point out that other choices are admissible. Our existence result still holds, with minor changes in the proof, for suitable Dirichlet, Robin, and nonhomogeneous Neumann boundary conditions, while the positivity of the (absolute) temperature should require some further compatibility properties. We finally remark that, for simplicity, most of the physical constants appearing in (1.12)-(1.14) will be set equal to 1 in the sequel.

The remainder of the paper is organized as follows. The next section deals with the notation, the assumptions, and the statement of the main result. In Sec. 3 we introduce a family of approximating problems through discretization and regularization procedures and prove their well-posedness. Next, Sec. 4 is devoted to the derivation of a rather unusual a priori estimate (independent of the approximation parameters) where the boundedness of $\partial_{t} \chi$ is a key point. In Sec. 5, we pass to the limit by using compactness and semicontinuity tools, recovering a solution of the original problem. Finally, Sec. 6 is 
concerned with the proof of the essential nonnegativity of the (absolute) temperature $\theta$, which is based on a standard maximum principle argument.

2. Main results. First of all, let us introduce some notation. Let $\Omega$ be a smooth and bounded domain of $\mathbb{R}^{d}(d \geq 1)$ and $T>0$ an assigned final time. We denote by $\Gamma$ the boundary of $\Omega$ and set $\Sigma:=\Gamma \times(0, T), Q_{t}:=\Omega \times(0, t)$, for $t \in(0, T]$, and $Q:=Q_{T}$. We also denote by $\mathbf{n}$ the outer normal unit vector to $\Omega$ at the points of $\Gamma$. Moreover, in the following we shall write $H:=L^{2}(\Omega), V:=H^{1}(\Omega)$ and identify $H$ with $H^{\prime}$, in order that the compact inclusions chain $V \subset H \subset V^{\prime}$ holds. The symbol $|\cdot|$ will stand for both the norms in $H$ and in $H^{d}$, and the notation $(\cdot, \cdot)$ will be used for the scalar product of $H$. Finally, we can set

$$
W:=\left\{v \in H^{2}(\Omega) \text { such that } \partial_{\mathbf{n}} v=0 \text { on } \Gamma\right\} ;
$$

clearly, we have that $W \subset V$ with compact inclusion.

We now start by listing the main mathematical hypotheses of our work. First, we introduce two convex, lower semicontinuous, and proper functions

$$
\phi, \psi: \mathbb{R} \rightarrow[0,+\infty),
$$

satisfying the following properties:

$$
0 \in D(\phi) \cap D(\psi), \quad \operatorname{int}(D(\phi))=(0, \lambda), \quad \phi(0)=\psi(0)=0,
$$

where $\lambda>0$ is an assigned constant accounting for the limit velocity of the phase transition process, and $D(\phi)$ (resp. $D(\psi)$ ) denotes the effective domain of $\phi$ (resp. $\psi$ ). We can now set $\alpha:=\partial \phi$ and $\beta:=\partial \psi$; then, $\alpha$ and $\beta$ can be seen as maximal monotone graphs in $\mathbb{R} \times \mathbb{R}$ (we refer to [5] for more details).

Moreover, we assume

$$
\begin{aligned}
& \theta_{c}>0 \quad \text { assigned constant, } \\
& \theta_{0} \in V, \quad 0 \leq \theta_{0} \quad \text { a.e. in } \Omega, \\
& \chi_{0} \in W, \quad \chi_{0} \in D(\beta) \text { a.e. in } \Omega, \\
& \text { there exists } \eta_{0} \in H, \text { such that } \eta_{0} \in \beta\left(\chi_{0}\right) \text { a.e. in } \Omega .
\end{aligned}
$$

Clearly, in the above positions, $D(\beta)$ denotes the domain of the graph $\beta$, i.e., $D(\beta)=$ $\{r \in \mathbb{R}: \beta(r) \neq \varnothing\}$; we recall that $D(\beta) \subset D(\psi)$.

We are now able to state the main result of this paper.

Theorem 2.1. There exists a quadruple $(\theta, \chi, \xi, \eta)$ of functions satisfying

$$
\begin{aligned}
& \theta \in H^{1}(0, T ; H) \cap C^{0}([0, T] ; V) \cap L^{2}(0, T ; W), \\
& \chi \in W^{1, \infty}(0, T ; H) \cap H^{1}(0, T ; V) \cap L^{\infty}(0, T ; W), \\
& \xi \in L^{\infty}(0, T ; H), \quad \eta \in L^{\infty}(0, T ; H), \\
& 0 \leq \theta \quad \text { and } \quad 0 \leq \partial_{t} \chi \leq \lambda \quad \text { a.e. in } Q,
\end{aligned}
$$


and such that the following equations hold a.e. in $Q$ :

$$
\begin{aligned}
& \partial_{t} \theta-\Delta \theta=\partial_{t} \chi\left(\partial_{t} \chi+\xi-\theta\right) \\
& \partial_{t} \chi+\xi-\Delta \chi+\eta=\theta-\theta_{c} \\
& \xi \in \alpha\left(\partial_{t} \chi\right) \text { and } \quad \eta \in \beta(\chi) .
\end{aligned}
$$

Moreover, the following initial conditions hold:

$$
\theta(x, 0)=\theta_{0}(x) \quad \text { and } \quad \chi(x, 0)=\chi_{0}(x) \text { a.e. in } \Omega .
$$

The proof of the above theorem will be carried out throughout all the remainder of the paper; for the present, we just give some further remarks about the structure of our system. Actually, as we already mentioned in the Introduction, the right-hand side of (2.12) can be rewritten by exploiting the structure of (2.13). Namely,

$$
\partial_{t} \theta-\Delta \theta=\partial_{t} \chi\left(\Delta \chi-\eta-\theta_{c}\right)
$$

Clearly, the couple (2.12)-(2.13) is absolutely equivalent to (2.16)-(2.13); hence, either structure of the energy balance equation will be indifferently used in the sequel.

Moreover, we point out that the highly nonlinear character of the system (2.12)(2.13) does not allow us to prove any uniqueness result, even in the case of $\beta$ Lipschitz continuous $[3,9]$. Indeed, although the global boundedness of $\partial_{t} \chi$ is guaranteed by the constraint $\alpha$, we are not able to estimate the product $\partial_{t} \chi \xi$ on the right-hand side of (2.12), which involves a multivalued operator. Even if the equivalent structure provided by (2.16) is chosen for the energy equation, a similar problem arises when one considers the term $\partial_{t} \chi \Delta \chi$.

3. Time discretization. We now introduce an approximate formulation of the system (2.12)-(2.15) and prove an existence result for such a regularized problem. Our method is essentially based on a backward time discretization. However, some preliminary work is needed. First of all, we have to substitute the graph $\beta$ in (2.13) by its Yosida regularization $\beta_{\varepsilon}$, where $\varepsilon$ is the approximation parameter. Referring to [5] for more details, we just recall here that $\beta_{\varepsilon}$ is a Lipschitz continuous graph. In addition, we denote by $A$ the realization in $H$ of the elliptic operator $-\Delta$ with Neumann boundary conditions simply given by

$$
A: W \rightarrow H, \quad A v:=-\Delta v \quad \forall v \in W .
$$

At this point, for any $N \in \mathbb{N}^{+}$, we subdivide the interval $[0, T]$ into $N$ subintervals of equal length, by letting $\tau:=T / N$; furthermore, we set $\theta^{0}:=\theta_{0}$, and $\chi^{0}:=\chi_{0}$, and consider the following scheme:

$$
\begin{gathered}
\frac{\theta^{i}-\theta^{i-1}}{\tau}+A \theta^{i}=\frac{\chi^{i}-\chi^{i-1}}{\tau}\left(-A \chi^{i}-\beta_{\varepsilon}\left(\chi^{i}\right)-\theta_{c}\right), \\
\frac{\chi^{i}-\chi^{i-1}}{\tau}+\xi^{i}+A \chi^{i}+\beta_{\varepsilon}\left(\chi^{i}\right)=\theta^{i-1}-\theta_{c}, \\
\xi^{i} \in \alpha\left(\frac{\chi^{i}-\chi^{i-1}}{\tau}\right) .
\end{gathered}
$$

We are in the position of proving an existence result for the above scheme. 
LEMmA 3.1. Under the above assumptions, there exists a triplet $\left\{\left(\theta^{i}, \chi^{i}, \xi^{i}\right)\right\}_{i=1}^{N}$ in $(W \times$ $W \times H)^{N}$ fulfilling relations (3.2)-(3.4) for $i=1, \ldots, N$.

Proof. We are going to proceed by induction on $i$. Indeed, we assume to know $\left(\theta^{i-1}, \chi^{i-1}\right) \in W^{2}$ (see $\left.(2.5)-(2.6)\right)$ and we look for $\left(\theta^{i}, \chi^{i}, \xi^{i}\right) \in(W \times W \times H)$. Our argument consists of the following two steps:

1. We consider the system (3.3)-(3.4) and observe that the right-hand side of (3.3) is known at level $i$. Then, we find a solution $\left(\chi^{i}, \xi^{i}\right) \in W \times H$ to (3.3)-(3.4).

2. Inserting the component $\chi^{i}$ of this solution into (3.2) and owing to the second condition of (2.3) and to the Lipschitz continuity of $\beta_{\varepsilon}$, we verify that the right-hand side of (3.2) is a known function of $H$. Thus, if we find $\theta^{i}$ satisfying (3.2), then it is immediate to check that the triplet $\left(\theta^{i}, \chi^{i}, \xi^{i}\right)$ solves (3.2)-(3.4).

Indeed, while the second step can be carried out by using the standard techniques for elliptic equations, the key point is 1., i.e., the resolution of (3.3)-(3.4) for any given $\left(\theta^{i-1}, \chi^{i-1}\right) \in W^{2}$. To this end, let us rewrite that system as the equivalent inclusion

$$
\tau \theta^{i-1}-\tau \theta_{c}+\chi^{i-1} \in \chi^{i}+\tau \alpha\left(\frac{\chi^{i}-\chi^{i-1}}{\tau}\right)+\tau\left(A \chi^{i}+\beta_{\varepsilon}\left(\chi^{i}\right)\right),
$$

where all the known terms have been collected on the left-hand side.

Actually, we would like to exploit the well-known results on the maximality of sums of monotone operators, holding under particular regularity conditions. Unfortunately, in order to fit the latter, also the graph $\alpha$ in (3.5) has to be regularized by its Yosida approximation $\alpha_{\sigma}$. Now, since $\chi^{i-1}$ is a datum, the operator

$$
\mathcal{B}_{\varepsilon, \sigma}: H \rightarrow H, \quad \mathcal{B}_{\varepsilon, \sigma}(v):=\tau \alpha_{\sigma}\left(\frac{v-\chi^{i-1}}{\tau}\right)+\tau \beta_{\varepsilon}(v), \quad \text { for } v \in H,
$$

is maximal monotone and Lipschitz continuous; in particular, its effective domain is the whole space $H$. Since $A$ is maximal monotone with domain $D(A)=W$, the hypotheses of, e.g., $\left[5\right.$, Cor. 2.7, p. 36] are fulfilled; hence, we find one solution $\chi_{\sigma}^{i}$ to the $\alpha_{\sigma}$-regularized version of (3.5).

Now, in order to remove the $\sigma$-approximation, we just test the $\alpha_{\sigma}$-regularized version of (3.5) by $(\mathrm{Id}+A)\left(\chi_{\sigma}^{i}-\chi^{i-1}\right)$, where Id stands for the identity in $H$. Denoting by $f^{i-1}$ the left-hand side of (3.5) and using the monotonicity of $\alpha_{\sigma}$ and the property $0=\alpha_{\sigma}(0)$, which is a consequence of (2.3), we easily infer

$$
\left(\chi_{\sigma}^{i}+\tau A \chi_{\sigma}^{i}+\tau \beta_{\varepsilon}\left(\chi_{\sigma}^{i}\right),(\operatorname{Id}+A)\left(\chi_{\sigma}^{i}-\chi^{i-1}\right)\right) \leq\left(f^{i-1},(\operatorname{Id}+A)\left(\chi_{\sigma}^{i}-\chi^{i-1}\right)\right) .
$$

Then, performing standard integrations by parts, using the monotonicity and Lipschitz continuity of $\beta_{\varepsilon}$, and denoting by $C_{i}$ a positive constant depending on $\tau, \varepsilon$, but not on $\sigma$, we deduce

$$
\left|\chi_{\sigma}^{i}\right|^{2}+(1+\tau)\left|\nabla \chi_{\sigma}^{i}\right|^{2}+\tau\left|A \chi_{\sigma}^{i}\right|^{2}\left|\chi_{\sigma}^{i}\right|^{2} \leq C_{i}\left(\left\|\chi^{i-1}\right\|_{W}^{2}+\left|f^{i-1}\right|^{2}\right) .
$$

From the above relation it immediately follows that, for some $\chi^{i} \in W$,

$$
\chi_{\sigma}^{i} \rightarrow \chi^{i} \quad \text { weakly in } W \text { and strongly in } V .
$$


Proceeding by comparison in the $\sigma$-regularization of $(3.5)$, we also derive that, for some $\xi^{i} \in H$

$$
\alpha_{\sigma}\left(\frac{\chi_{\sigma}^{i}-\chi^{i-1}}{\tau}\right) \rightarrow \xi^{i} \quad \text { weakly in } H .
$$

Then, (3.4) is a consequence of the usual monotonicity argument of [2, Prop. 1.1, p. 42], and this completes the proof that $\left(\theta^{i}, \chi^{i}, \xi^{i}\right)$ solves $(3.2)-(3.4)$.

REMARK 3.2. We observe that it is essential to remove the $\sigma$-approximation at the discretized level. Indeed, in the subsequent passage to the limit with respect to the approximation parameters $\varepsilon$ and $\tau$, we shall strongly exploit the global boundedness of $\left(\chi^{i}-\chi^{i-1}\right) / \tau$, which is ensured by the presence of the constraining original graph $\alpha$.

4. A priori estimates. In this section we aim to establish some a priori estimates on the time-discrete solutions whose existence has been proved above. For the sake of clarity, we introduce some notation. Let $\left\{u_{i}\right\}, i=0,1, \ldots, N$, be a vector. Then, we denote by $u_{\tau}$ and $\bar{u}_{\tau}$ two functions of the time interval $[0, T]$ that interpolate the values of the vector piecewise linearly and backward constantly, respectively. That is,

$$
\begin{gathered}
u_{\tau}(0):=u_{0}, \quad u_{\tau}(t):=a_{i}(t) u_{i}+\left(1-a_{i}(t)\right) u_{i-1}, \\
\bar{u}_{\tau}(0):=u_{0}, \quad \bar{u}_{\tau}(t):=u_{i}, \quad \text { for } t \in((i-1) \tau, i \tau], \quad i=1, \ldots, N,
\end{gathered}
$$

where $a_{i}(t):=(t-(i-1) \tau) / \tau$ for $t \in((i-1) \tau, i \tau], i=1, \ldots, N$. Moreover, let us introduce the translation operator $\mathcal{T}_{\tau}$ related to the time step $\tau$ by setting

$$
\begin{gathered}
\left(\mathcal{T}_{\tau} u\right)(t):=u(0) \quad \forall t \in[0, \tau) \quad \text { and } \quad\left(\mathcal{T}_{\tau} u\right)(t):=u(t-\tau) \quad \forall t \in[\tau, T], \\
\forall u:[0, T] \rightarrow \mathbb{R} .
\end{gathered}
$$

Owing to the position (4.1), the scheme (3.2)-(3.4) can be restated as

$$
\begin{gathered}
\partial_{t} \theta_{\tau}+A \bar{\theta}_{\tau}=\partial_{\tau} \chi_{\tau}\left(-A \bar{\chi}_{\tau}-\bar{\eta}_{\tau}-\theta_{c}\right), \\
\partial_{t} \chi_{\tau}+\bar{\xi}_{\tau}+A \bar{\chi}_{\tau}+\bar{\eta}_{\tau}=\mathcal{T}_{\tau} \bar{\theta}_{\tau}-\theta_{c}, \\
\bar{\xi}_{\tau} \in \alpha\left(\partial_{t} \chi_{\tau}\right), \quad \bar{\eta}_{\tau}=\beta_{\varepsilon}\left(\bar{\chi}_{\tau}\right) .
\end{gathered}
$$

Let us point out the introduction in (4.3)-(4.4) of the auxiliary vector

$$
\eta^{i}:=\beta_{\varepsilon}\left(\chi^{i}\right) \text { for } i=0,1, \ldots, N,
$$

and observe that, from the basic properties of the Yosida approximation of $\beta$, it easily follows that

$$
\left|\eta^{0}\right| \leq\left|\eta_{0}\right| \quad \forall \varepsilon>0
$$

The above notation (4.1) refers only to $\tau$ and not to $\varepsilon$ as one would expect. This apparently misleading choice causes no confusion and is motivated by the sake of clarity. Indeed, as will be evident in the next section, we will pass to the limit simultaneously in both $\tau$ and $\varepsilon$. Namely, we aim to consider some $\varepsilon=\varepsilon(\tau)$ vanishing indeed as $\tau \rightarrow 0$. In this concern, relations (4.2)-(4.4) represent an approximation scheme also for the original problem (2.12)-(2.14) (not only for the regularized problem with $\beta_{\varepsilon}$ in place of $\beta$ ). 
We are now in the position of proving our estimates. First of all, note that we obviously have $\partial_{t} \chi_{\tau} \in D(\alpha)$ a.e. in $Q$; thus

$$
0 \leq \partial_{t} \chi_{\tau} \leq \lambda \text { a.e. in } Q .
$$

Actually, this bound turns out to be critical for the sequel.

We begin by multiplying Eq. (3.2) by $\tau \delta \theta^{i}$ where $\delta \theta^{i}:=\left(\theta^{i}-\theta^{i-1}\right) / \tau$ and integrating on $\Omega$. Letting also $\delta \chi^{i}:=\left(\chi^{i}-\chi^{i-1}\right) / \tau$ we obtain

$$
\tau\left|\delta \theta^{i}\right|^{2}+\tau\left(A \theta^{i}, \delta \theta^{i}\right)=-\tau \int_{\Omega} \delta \chi^{i}\left(A \chi^{i}+\eta^{i}\right) \delta \theta^{i}-\tau \int_{\Omega} \delta \chi^{i} \theta_{c} \delta \theta^{i} .
$$

It is now straightforward to deduce that

$$
\tau\left(A \theta^{i}, \delta \theta^{i}\right)=\frac{1}{2}\left(\left|\nabla \theta^{i}\right|^{2}+\left|\nabla\left(\theta^{i}-\theta^{i-1}\right)\right|^{2}-\left|\nabla \theta^{i-1}\right|^{2}\right) .
$$

Moreover, the right-hand side of (4.8) may easily be controlled by virtue of (4.7) as follows:

$$
\begin{aligned}
-\tau \int_{\Omega} \delta \chi^{i}\left(A \chi^{i}+\eta^{i}\right) \delta \theta^{i} & \leq \tau \lambda\left|A \chi^{i}+\eta^{i}\right|\left|\delta \theta^{i}\right| \\
& \leq \frac{\tau}{4}\left|\delta \theta^{i}\right|^{2}+\tau \lambda^{2}\left|A \chi^{i}+\eta^{i}\right|^{2}-\tau \int_{\Omega} \delta \chi^{i} \theta_{c} \delta \theta^{i} \\
& \leq \frac{\tau}{4}\left|\delta \theta^{i}\right|^{2}+\tau \lambda^{2} \theta_{c}^{2}|\Omega|
\end{aligned}
$$

where $|\Omega|$ stands for the Lebesgue measure of $\Omega$. Now, by taking the sum of relation (4.8) for $i=1, \ldots, m(m \leq N)$, we get

$$
\begin{aligned}
& \frac{1}{2} \sum_{i=1}^{m} \tau\left|\delta \theta^{i}\right|^{2}+\frac{1}{2}\left|\nabla \theta^{m}\right|^{2}+\frac{1}{2} \sum_{i=1}^{m}\left|\tau \nabla \delta \theta^{i}\right|^{2} \\
& \quad \leq \frac{1}{2}\left|\nabla \theta_{0}\right|^{2}+\lambda^{2} \sum_{i=1}^{m} \tau\left|A \chi^{i}+\eta^{i}\right|^{2}+m \tau \lambda^{2} \theta_{c}^{2}|\Omega| .
\end{aligned}
$$

On the other hand, we can multiply relation (3.3) by $\tau\left(A \delta \chi^{i}+\delta \eta^{i}\right)$, where $\delta \eta^{i}:=$ $\left(\eta^{i}-\eta^{i-1}\right) / \tau$, and integrate on $\Omega$, obtaining

$$
\begin{aligned}
\tau\left|\nabla \delta \chi^{i}\right|^{2}+\tau\left(\delta \chi^{i}, \delta \eta^{i}\right)+ & \tau\left(A \chi^{i}+\eta^{i}, A \delta \chi^{i}+\delta \eta^{i}\right) \\
& +\tau\left(\xi^{i}, \delta A \chi^{i}\right)+\tau\left(\xi^{i}, \delta \eta^{i}\right) \leq \tau\left(\theta^{i-1}-\theta_{c}, A \delta \chi^{i}+\delta \eta^{i}\right)
\end{aligned}
$$

As before, we readily infer that

$$
\begin{aligned}
\tau\left(A \chi^{i}+\right. & \left.\eta^{i}, A \delta \chi^{i}+\delta \eta^{i}\right) \\
& =\frac{1}{2}\left(\left|A \chi^{i}+\eta^{i}\right|^{2}+\left|\left(A \chi^{i}+\eta^{i}\right)-\left(A \chi^{i-1}+\eta^{i-1}\right)\right|^{2}-\left|A \chi^{i-1}+\eta^{i-1}\right|^{2}\right)
\end{aligned}
$$

Moreover, the relation

$$
\tau\left(\delta \chi^{i}, \delta \eta^{i}\right) \geq 0
$$

is ensured by (4.5) and the monotonicity of $\beta_{\varepsilon}$, while

$$
\tau\left(\xi^{i}, A \delta \chi^{i}\right) \geq 0
$$

is a consequence of the following Lemma 4.1 concerning maximal monotone operators. This result justifies rigorously a formal monotonicity argument that frequently occurs in 
the derivation of the a priori estimates for this kind of problem. We include its proof for the sake of completeness.

LEMmA 4.1. Let $\gamma$ be a maximal monotone graph in $\mathbb{R} \times \mathbb{R}, A: W \rightarrow H$ as in (3.1), $u \in W, \zeta \in H$ such that $\zeta \in \gamma(u)$ a.e. in $\Omega$. Then, we have that

$$
\int_{\Omega} A u \zeta d x \geq 0
$$

Proof. Denoting by $\gamma_{\varepsilon}$ the Yosida approximation of $\gamma$, we consider the following elliptic problem:

$$
u_{\varepsilon}+A u_{\varepsilon}+\gamma_{\varepsilon}\left(u_{\varepsilon}\right)=u+A u+\zeta \text { in } H
$$

admitting, of course, a unique solution $u_{\varepsilon} \in W$. Since $\gamma_{\varepsilon}$ is Lipschitz continuous, there are no difficulties in deriving the natural a priori estimates for the above problem, leading to the following convergences, which hold, for some $v \in V$, up to the extraction of subsequences (actually uniqueness will guarantee them for the whole sequences):

$$
\begin{aligned}
u_{\varepsilon} \rightarrow v & \text { weakly in } V \text { and strongly in } H, \\
A u_{\varepsilon} \rightarrow A v & \text { weakly in } H \\
\gamma_{\varepsilon}\left(u_{\varepsilon}\right) \rightarrow \eta & \text { weakly in } H .
\end{aligned}
$$

Then, we see that the limit functions satisfy $v+A v+\eta=u+A u+\zeta$; if we show that $\eta \in \gamma(v)$ a.e. in $\Omega$, then, using again [2, Prop. 1.1, p. 42], we can conclude that $v=u$ and $\zeta=\eta$.

We easily deduce from (4.13) that

$$
\left(\gamma_{\varepsilon}\left(u_{\varepsilon}\right), u_{\varepsilon}\right)=\left(u+A u+\zeta-u_{\varepsilon}-A u_{\varepsilon}, u_{\varepsilon}\right),
$$

whence $\lim _{\varepsilon \rightarrow 0}\left(\gamma_{\varepsilon}\left(u_{\varepsilon}\right), u_{\varepsilon}\right)=(u+A u+\zeta-v-A v, v)=(\eta, v)$, as desired. We finally verify the required property. At the level $\varepsilon>0$, we have

$$
0 \leq \int_{\Omega} A u_{\varepsilon} \gamma_{\varepsilon}\left(u_{\varepsilon}\right) d x=\int_{\Omega}\left(u+A u+\zeta-u_{\varepsilon}-\gamma_{\varepsilon}\left(u_{\varepsilon}\right)\right) \gamma_{\varepsilon}\left(u_{\varepsilon}\right) d x
$$

so that, passing to the lim sup and exploiting semicontinuity, we infer

$$
0 \leq \int_{\Omega}(u+A u+\zeta-u-\zeta) \zeta d x=\int_{\Omega} A u \zeta d x
$$

Our next aim is to control the last term on the left-hand side of (4.10). Indeed, it is a standard matter to check that

$$
\tau\left(\xi^{i}, \delta \eta^{i}\right)=\tau \int_{\omega} \xi^{i} \delta \eta^{i} \frac{\delta \chi^{i}}{\delta \chi^{i}} \geq 0
$$

where $\omega=\left\{\delta \chi^{i} \neq 0\right\}$ and we exploited the monotonicity of $\alpha$ and $\beta_{\varepsilon}$, together with $0 \in \alpha(0)$. 
Next, we make use of the above considerations and take the sum in (4.10) as $i=$ $1, \ldots, m(m \leq N)$. One has

$$
\begin{gathered}
\sum_{i=1}^{m} \tau\left|\nabla \delta \chi^{i}\right|^{2}+\frac{1}{2}\left|A \chi^{m}+\eta^{m}\right|^{2}+\frac{1}{2} \sum_{i=1}^{m}\left|\tau\left(A \delta \chi^{i}+\delta \eta^{i}\right)\right|^{2} \\
\leq \frac{1}{2}\left|A \chi_{0}+\eta^{0}\right|^{2}+\sum_{i=1}^{m} \tau\left(\theta^{i-1}-\theta_{c}, A \delta \chi^{i}+\delta \eta^{i}\right) .
\end{gathered}
$$

The second term on the right-hand side above may be handled by means of a discrete integration by parts procedure. Namely, we easily infer that

$$
\begin{aligned}
\sum_{i=1}^{m} \tau\left(\theta^{i-1}\right. & \left.-\theta_{c}, A \delta \chi^{i}+\delta \eta^{i}\right) \\
& =-\sum_{i=1}^{m-1} \tau\left(\delta \theta^{i}, A \chi^{i}+\eta^{i}\right)+\left(\theta^{m-1}-\theta_{c}, A \chi^{m}+\eta^{m}\right)-\left(\theta_{0}-\theta_{c}, A \chi_{0}+\eta^{0}\right) .
\end{aligned}
$$

Moreover, plain calculations lead to the following:

$$
\begin{aligned}
\left|\theta^{m-1}\right|^{2} & \leq 2\left|\theta_{0}\right|^{2}+2\left(\sum_{i=1}^{m-1} \tau\left|\delta \theta^{i}\right|\right)^{2} \\
& \leq 2\left|\theta_{0}\right|^{2}+2(m-1) \tau \sum_{i=1}^{m-1} \tau\left|\delta \theta^{i}\right|^{2} .
\end{aligned}
$$

Whence, looking back to (4.6) and (4.19), it is not difficult to find a positive constant $C_{1}$ that depends only on data, in particular, on $\left|\theta_{0}\right|, \theta_{c},\left\|\chi_{0}\right\|_{W},\left|\eta_{0}\right|$, and $T$, such that the following estimate holds:

$$
\begin{gathered}
\sum_{i=1}^{m} \tau\left|\nabla \delta \chi^{i}\right|^{2}+\frac{1}{4}\left|A \chi^{m}+\eta^{m}\right|^{2}+\frac{1}{2} \sum_{i=1}^{m}\left|\tau\left(A \delta \chi^{i}+\delta \eta^{i}\right)\right|^{2} \\
\leq C_{1}\left(1+\sum_{i=1}^{m-1} \tau\left(\frac{\left|\delta \theta^{i}\right|^{2}}{4}+\left|A \chi^{i}+\eta^{i}\right|^{2}\right)\right) .
\end{gathered}
$$

Finally, we multiply (4.9) by $C_{1}$ and add it to the inequality above, obtaining

$$
\begin{aligned}
\frac{C_{1}}{4} \sum_{i=1}^{m} \tau\left|\delta \theta^{i}\right|^{2} & +\frac{C_{1}}{2}\left|\nabla \theta^{m}\right|^{2}+\frac{C_{1}}{2} \sum_{i=1}^{m}\left|\tau \nabla \delta \theta^{i}\right|^{2} \\
& +\sum_{i=1}^{m} \tau\left|\nabla \delta \chi^{i}\right|^{2}+\frac{1}{4}\left|A \chi^{m}+\eta^{m}\right|^{2}+\frac{1}{2} \sum_{i=1}^{m}\left|\tau\left(A \delta \chi^{i}+\delta \eta^{i}\right)\right|^{2} \\
& \leq C_{1}\left(1+T \lambda^{2} \theta_{c}^{2}|\Omega|+\frac{1}{2}\left|\nabla \theta_{0}\right|^{2}+\left(\lambda^{2}+1\right) \sum_{i=1}^{m} \tau\left|A \chi^{i}+\eta^{i}\right|^{2}\right) .
\end{aligned}
$$

Whence, upon choosing the time step $\tau$ small enough (namely $\tau<\left(4 C_{1}\left(\lambda^{2}+1\right)\right)^{-1}$ ), we are in the position of applying the discrete Gronwall lemma (see, e.g., the version reported 
in [11, Prop. 2.2.1]). Accounting also for (4.7), the monotonicity of $\beta_{\varepsilon}$, standard elliptic arguments, and the notation (4.1), we may eventually establish the following bounds:

$$
\begin{aligned}
\left\|\partial_{t} \theta_{\tau}\right\|_{L^{2}(0, T ; H)},\left\|\bar{\theta}_{\tau}\right\|_{L^{\infty}(0 . T: V)},\left\|\partial_{t} \chi_{\tau}\right\|_{L^{2}(0, T ; V) \cap L^{\infty}(Q)}, \\
\left\|\bar{\chi}_{\tau}\right\|_{L^{\infty}(0, T ; W)},\left\|\bar{\eta}_{\tau}\right\|_{L^{\infty}(0, T ; H)} \leq C_{2}
\end{aligned}
$$

for a proper constant $C_{2}$ depending on data but independent of both $\tau$ and $\varepsilon$. Owing to (4.21), it is straightforward to obtain by a comparison in (4.2) and (4.3) that

$$
\left\|\bar{\theta}_{\tau}\right\|_{L^{2}(0, T ; W)},\left\|\bar{\xi}_{\tau}\right\|_{L^{\infty}(0, T ; H)} \leq C_{3}
$$

where $C_{3}$ has the same dependences as $C_{2}$.

Before passing to the limit, let us point out some useful estimates on the difference of approximate solutions. Indeed, it is straightforward to verify that

$$
\begin{gathered}
\left\|\theta_{\tau}-\bar{\theta}_{\tau}\right\|_{L^{2}(0, T ; H)},\left\|\chi_{\tau}-\bar{\chi}_{\tau}\right\|_{L^{2}(0, T ; V)} \leq C_{2} \tau \\
\left\|\theta_{\tau}-\bar{\theta}_{\tau}\right\|_{L^{\infty}(0, T ; H)},\left\|\bar{\theta}_{\tau}-\mathcal{T}_{\tau} \bar{\theta}_{\tau}\right\|_{L^{\infty}(0, T ; H)},\left\|\chi_{\tau}-\bar{\chi}_{\tau}\right\|_{L^{\infty}(0, T ; V)} \leq C_{2} \sqrt{\tau}
\end{gathered}
$$

as a direct check provides.

REMARK 4.2. For the sake of numerical purposes, it is worth noting that the above detailed time discretization procedure may be easily extended to variable time step partitions of the interval $[0, T]$. Although the approximation argument developed here serves perfectly to the aim of proving Theorem 2.1, we stress that this analysis may be easily reproduced for a partition

$$
\mathcal{P}=\left\{0=t_{0}<t_{1}<\cdots<t_{N-1}<t_{N}=T\right\}
$$

with a variable time step $\tau_{i}:=t_{i}-t_{i-1}$ for $i=1, \ldots, N$ and a diameter $\tau:=\max _{1 \leq i \leq N} \tau_{i}$. From this point of view, our procedure seems to have an additional numerical interest. Indeed, the only constraint imposed to the time step sizes is $\tau<\left(4 C_{1}\left(\lambda^{2}+1\right)\right)^{-1}$. Thus, the latter time steps may be chosen adaptively, being possibly tailored to some further numerical consideration.

5. Passage to the limit. In order to prove Theorem 2.1, we aim to pass to the limit simultaneously with respect to both of the approximation parameters $\varepsilon$ and $\tau$ in relations (4.2)-(4.4). Namely, we set

$$
\varepsilon=\varepsilon(\tau) \text { such that } \varepsilon(\tau) \rightarrow 0 \text { as } \tau \rightarrow 0 \text {. }
$$

In this setting, all the forthcoming passage-to-the-limit procedures will obviously refer just to $\tau$. Thanks to well-known compactness results, estimates (4.21)-(4.24), and easy 
considerations, we may find a quadruple $(\theta, \chi, \eta, \xi)$ such that, possibly taking subsequences (not relabelled), one has

$$
\begin{gathered}
\theta_{\tau} \rightarrow \theta \quad \text { weakly star in } H^{1}(0, T ; H) \cap L^{\infty}(0, T ; V) \cap L^{2}(0, T ; W), \\
\bar{\theta}_{\tau} \rightarrow \theta \quad \text { weakly star in } L^{\infty}(0, T ; V) \cap L^{2}(0, T ; W), \\
\chi_{\tau} \rightarrow \chi \quad \text { weakly star in } H^{1}(0, T ; V) \cap L^{\infty}(0, T ; W), \\
\partial_{t} \chi_{\tau} \rightarrow \partial_{t} \chi \quad \text { weakly star in } L^{\infty}(Q), \\
\bar{\chi}_{\tau} \rightarrow \chi \quad \text { weakly star in } L^{\infty}(0, T ; W), \\
\bar{\eta}_{\tau} \rightarrow \eta \quad \text { weakly star in } L^{\infty}(0, T ; H), \\
\bar{\xi}_{\tau} \rightarrow \xi \quad \text { weakly star in } L^{\infty}(0, T ; H)
\end{gathered}
$$

Clearly, we can consider the limit $\theta$ (resp. $\chi$ ) of either $\theta_{\tau}$ or $\bar{\theta}_{\tau}$ (resp. $\chi_{\tau}$ or $\bar{\chi}_{\tau}$ ), thanks to (4.23)-(4.24). Moreover, by the generalized Ascoli theorem (see, e.g., [14, Cor. 4]) and (4.24), the following convergences may also be inferred:

$$
\begin{aligned}
\theta_{\tau} \rightarrow \theta & \text { strongly in } C^{0}([0, T] ; H), \\
\bar{\theta}_{\tau} \rightarrow \theta & \text { strongly in } L^{\infty}(0, T ; H), \\
\mathcal{T}_{\tau} \bar{\theta}_{\tau} \rightarrow \theta & \text { strongly in } L^{\infty}(0, T ; H), \\
\chi_{\tau} \rightarrow \chi & \text { strongly in } C^{0}([0, T] ; V), \\
\bar{\chi}_{\tau} \rightarrow \chi & \text { strongly in } L^{\infty}(0, T ; V) .
\end{aligned}
$$

Hence, we can pass to the limit in (4.3), and, furthermore, we see that the properties (2.8)-(2.10) along with (2.13) are fulfilled by the quadruple $(\theta, \chi, \eta, \xi)$.

Now we address the interpretation of $\eta$ and $\xi$. As for $\eta$, we observe that relations (5.6) and $(5.12)$ allow us to use [2, Prop. 1.1, p. 42], which readily yields the second condition of (2.14). If we were able to prove that

$$
\limsup _{\tau \rightarrow 0} \iint_{Q} \bar{\xi}_{\tau} \partial_{t} \chi_{\tau} \leq \iint_{Q} \xi \partial_{t} \chi
$$

we might apply again [2, Prop. 1.1, p. 42] and the first condition of (2.14) (and, obviously, the second one of $(2.11)$ ) would be satisfied as well.

Towards the aim of proving the above inequality, we multiply (4.3) by $\partial_{t} \chi_{\tau}$ and integrate over $Q$, obtaining

$$
\begin{aligned}
\iint_{Q} \bar{\xi}_{\tau} \partial_{t} \chi_{\tau}=-\iint_{Q}\left(\partial_{t} \chi_{\tau}\right)^{2}-\iint_{Q} \nabla \bar{\chi}_{\tau} \cdot \nabla \partial_{t} \chi_{\tau} & \\
& -\iint_{Q} \bar{\eta}_{\tau} \partial_{t} \chi_{\tau}+\iint_{Q}\left(\mathcal{T}_{\tau} \bar{\theta}_{\tau}-\theta_{c}\right) \partial_{t} \chi_{\tau}
\end{aligned}
$$

Next, we take the limsup of both sides of (5.14) as $\tau$ goes to 0 . We remark that $\overline{\eta_{\tau}}=\beta_{\varepsilon}\left(\bar{\chi}_{\tau}\right)=\partial \psi_{\varepsilon}\left(\bar{\chi}_{\tau}\right)$ (recall that $\beta=\partial \psi$ ), where $\psi_{\varepsilon}$ denotes the Yosida approximation 
of $\psi$. Then, we integrate in time and, using, e.g., [5, Lemma 3.3, p. 73], we get

$$
\begin{aligned}
\limsup _{\tau \rightarrow 0}-\iint_{Q} \bar{\eta}_{\tau} \partial_{t} \chi_{\tau} & =-\liminf _{\tau \rightarrow 0}\left(\int_{\Omega} \psi_{\varepsilon}\left(\bar{\chi}_{\tau}(T)\right) d x-\int_{\Omega} \psi_{\varepsilon}\left(\chi_{0}\right) d x\right) \\
& \leq-\int_{\Omega} \psi(\chi(T)) d x+\int_{\Omega} \psi\left(\chi_{0}\right) d x
\end{aligned}
$$

and the last inequality holds since the functional induced by $\psi_{\varepsilon}$ on $H$, namely,

$$
\Psi_{\varepsilon}(v):=\int_{\Omega} \psi_{\varepsilon}(v) d x \quad \text { for } v \in H,
$$

converges to

$$
\Psi(v):= \begin{cases}\int_{\Omega} \psi(v) d x & \text { if } v \in H \text { and } \psi(v) \in L^{1}(\Omega), \\ +\infty & \text { otherwise }\end{cases}
$$

in the sense of Mosco [1, Prop. 3.56, p. 354] in $H$, and (5.11) holds.

Owing to (5.3), (5.12), (5.15), and (5.10), and comparing the limit of (5.14) with (2.13), we derive relation (5.13) and also the first condition in (2.14) is proved. Moreover, according to $[2$, Lemma 1.3 , p. 42$]$, we also have

$$
\iint_{Q} \bar{\xi}_{\tau} \partial_{t} \chi_{\tau} \rightarrow \iint_{Q} \xi \partial_{t} \chi \quad \text { as } \tau \rightarrow 0 .
$$

Finally, we pass to the limit in (4.2). As usual, let us rewrite it as

$$
\partial_{t} \theta_{\tau}+A \bar{\theta}_{\tau}=\partial_{t} \chi_{\tau}\left(\partial_{t} \chi_{\tau}+\bar{\xi}_{\tau}-\mathcal{T}_{\tau} \bar{\theta}_{\tau}\right)
$$

clearly, on account of the above argument, we only need some strong convergence of $\partial_{t} \chi_{\tau}$. Actually, it is sufficient to multiply (4.3) again by $\partial_{t} \chi_{\tau}$, to integrate over $Q$, and to take the limsup. Using, in particular, (5.18) and comparing with (2.13), we get that

$$
\limsup _{\tau \rightarrow 0} \iint_{Q}\left(\partial_{t} \chi_{\tau}\right)^{2} \leq \iint_{Q}\left(\partial_{t} \chi\right)^{2}
$$

which, together with (5.3) implies that

$$
\partial_{t} \chi_{\tau} \rightarrow \partial_{t} \chi \quad \text { strongly in } L^{2}(0, T ; H)
$$

This is enough to pass to the limit in (5.19), showing indeed that our solution satisfies (2.12) as well.

6. Positivity of the temperature. We conclude the proof of Theorem 2.1 by establishing the essential nonnegativity of the temperature $\theta$. To this aim, we are going to prove a suitable maximum principle. Indeed, we multiply Eq. (2.12) by the function

$$
-(\theta)^{-}:=\min \{0, \theta\} \in L^{2}(0, T ; H),
$$

integrate on $Q_{t}, t \in(0, T]$ and get that

$$
\frac{1}{2}\left|\theta^{-}(t)\right|^{2}+\int_{0}^{t}\left|\nabla\left(\theta^{-}\right)(s)\right|^{2} d s \leq \sum_{j=1}^{4} I_{j}(t),
$$


where

$$
\begin{aligned}
I_{1} & :=\frac{1}{2}\left|\theta^{-}(0)\right|^{2}=\frac{1}{2}\left|\theta_{0}^{-}\right|^{2}, \\
I_{2}(t) & :=-\int_{0}^{t} \int_{\Omega}\left(\partial_{t} \chi\right)^{2} \theta^{-} d x d s, \\
I_{3}(t) & :=-\int_{0}^{t} \int_{\Omega} \partial_{t} \chi \xi \theta^{-} d x d s, \\
I_{4}(t) & :=\int_{0}^{t} \int_{\Omega} \partial_{t} \chi \theta \theta^{-} d x d s .
\end{aligned}
$$

Since (2.5) holds, we readily get that $I_{1}=0$. Moreover, the term $I_{2}(t)$ turns out to be obviously nonpositive for all $t>0$. Then, owing to (2.3) and (2.14), we easily deduce that $\partial_{t} \chi \xi \geq 0$ a.e. in $Q$. Namely, we have that $\xi \leq 0$ if and only if $\partial_{t} \chi$ vanishes; thus also $I_{3}(t)$ is nonpositive for all $t>0$. As regards $I_{4}(t)$, it is a standard matter to check that $\partial_{t} \chi \theta \theta^{-}=-\partial_{t} \chi\left(\theta^{-}\right)^{2} \leq 0$ a.e. in $Q$. Collecting all the above considerations, one has that the right-hand side of $(6.1)$ is nonpositive. Hence, we infer that

$$
\left|\theta^{-}(t)\right|^{2}=0 \quad \forall t \in(0, T)
$$

and relation (2.11) is completely proved. This concludes the proof of Theorem 2.1.

REMARK 6.1. Let us point out that the nonnegativity of the discrete temperature $\bar{\theta}_{\tau}$ may not be deduced from a time discrete analogue to the argument above. Indeed, this possibility fails since the term

$$
I_{4}(i \tau)=\int_{0}^{i \tau} \partial_{t} \chi_{\tau} \mathcal{T}_{\tau} \bar{\theta}_{\tau}\left(\bar{\theta}_{\tau}\right)^{-}
$$

which corresponds to $I_{4}(t)$ above, turns out to be nonnegative whenever we ask for $\bar{\theta}_{\tau} \geq 0$ a.e. in $\Omega \times(0,(i-1) \tau)$ in the framework of an induction proof.

\section{REFERENCES}

[1] H. Attouch, Variational Convergence for Functions and Operators, Pitman, London, 1984

[2] V. Barbu, Nonlinear Semigroups and Differential Equations in Banach Spaces, Noordhoff, Leyden, 1976

[3] G. Bonfanti, M. Frémond, and F. Luterotti, Global solution to a nonlinear system for irreversible phase changes, Adv. Math. Sci. Appl. 10, 1-24 (2000)

[4] G. Bonfanti, M. Frémond, and F. Luterotti, Local solutions to the full model of phase transitions with dissipation, Adv. Math. Sci. Appl. 11, 791-810 (2001)

[5] H. Brezis, Opérateurs Maximaux Monotones et Sémi-groupes de Contractions dans les Espaces de Hilbert, North-Holland Math. Studies, vol. 5, North-Holland, Amsterdam, 1973

[6] G. Caginalp, An analysis of a phase field model of a free boundary, Arch. Rational Mech. Anal. 92, 205-245 (1986)

[7] G. Caginalp and X. Chen, Convergence of the phase field model to its sharp interface limits, European J. Appl. Math. 9, 417-445 (1998)

[8] P. Colli, M. Frémond, and O. Klein, Global existence of a solution to phase field model for supercooling, Nonlinear Anal. Real World Appl. 2, 523-539 (2001)

[9] P. Colli, F. Luterotti, G. Schimperna, and U. Stefanelli, Global existence for a class of generalized systems for irreversible phase changes, NoDEA Nonlinear Differential Equations Appl., 2001, to appear

[10] M. Frémond and A. Visintin, Dissipation dans le changement de phase. Surfusion. Changement de phase irreversible, C. R. Acad. Sci. Paris Sér. II Méc. Phys. Chim. Sci. Univers. Sci. Terre 301, 1265-1268 (1985) 
[11] J. W. Jerome, Approximation of nonlinear evolution systems, Ser. Math. Sci. Engrg., no. 164, Academic Press, Orlando, 1983

[12] F. Luterotti, G. Schimperna, and U. Stefanelli, Existence result for a nonlinear model related to irreversible phase changes, $\mathrm{M}^{3}$ AS, Math. Models Methods Appl. Sci. 11, 809-825 (2001)

[13] F. Luterotti and U. Stefanelli, One dimensional results to the full model of phase transitions, Z. Anal. Anwendungen, 2001, to appear

[14] J. Simon, Compact sets in the space $L^{p}(0, T ; B)$, Ann. Mat. Pura Appl. (4), 146, 65-96 (1987) 